RNA yet identified to act in trans as a true ribozyme without prior modification.

The detailed reaction mechanisms and structural manipulations of group I and II self-slicing introns still present many challenges, as discussed here. But there has been considerable progress in other areas, such as the characterization of small, circular, single-stranded pathogenic RNAs of plants. Earlier work indicated that they are replicated in vivo by a rolling-circle mechanism in which the circular RNAs are copied to produce multimeric intermediates that are then processed to monomeric units. Attempts to find protein-free, RNA-catalysed processing reactions in some of these plant pathogenic RNAs were successful, and so were born the hammerhead and hairpin ribozymes.

The small size of the hammerhead ribozyme allows its easy manipulation. Its sequence can be split into ribozyme and substrate components so that cleavage could occur in trans, and it has been extensively demonstrated that the ribozyme component of the hammerhead can be designed to target and cleave foreign messenger and other RNAs in vitro. There are many studies of the cleavage of foreign RNAs, and on the effort to use the in trans reaction in vivo for control of intracellular reactions and pathogen infections, but it is still to be determined whether these will be more effective than antisensense approaches. Hence it is not surprising that the hammerhead ribozyme dominates this volume, especially as it is also the area of research interest of the two editors.

They have included the three-dimensional crystalline structure of the hammerhead ribozyme, first reported in 1994, and the development of in vitro selection techniques in the search for new types of ribozymes. However, we need to ask how many naturally occurring ribozymes are yet to be discovered. Initially the small, circular, plant pathogenic RNAs were a good place to start looking, in view of the discovery of the hammerhead and hairpin ribozymes. Plant viroids are also likely candidates, as two of these, avocado sunblotch viroid and peach latent mosaic viroid, show hammerhead self-cleavage in the plus and minus strands. The lack of success in finding any RNA-catalysed reactions in the other viroids has led some researchers to believe that the processing of multimeric intermedi- ates is catalysed by protein rather than RNA. I think it is far more likely that ribozymes will be found when we have developed the appropriate experimental approaches to search for them.

This volume is a rich source of information on catalytic RNA, with many of the major players contributing articles. The only obvious omissions are the as yet uncharacterized ribozyme that is found in an 881nucleotide transcript of a circular Neurospo$r a$ mitochondrial plasmid, and the extensively characterized ribozyme of hepatitis delta virus, the only ribozyme so far identified in animal systems.

Bob Symons is in the Department of Plant Science, Waite Agricultural Research Institute, University of Adelaide, Glen Osmond, South Australia 5064, Australia.

\section{Spring books supplement}

The 27 March issue of Nature will contain the Spring Books supplement. Reviewers are likely to include Walter Gratzer on the discovery of the structure of DNA, David Jones (perhaps better known to Nature readers as Daedalus) on design, and Sir Crispin Tickell on T. H. Huxley.

\title{
Specialist books
}

Excellent $x+x+$ Good $t+x+$ Average

\section{Relativity and Gravitation}

by Philippe Tourrenc

Cambridge University Press: 1996. Pp. 242. £45,

$\$ 74.95$ ( $h b k$ ); $£ 16.95, \$ 29.95$ (pbk)

The nature of gravity has intrigued physicists for centuries, and progress in our understanding has required major changes in our view of the world. With a clearly stated view of the philosophical divide between the newtonian and relativistic worlds, Philippe Tourrenc gives a wide-ranging introduction to special and general relativity.

With discussions of applications, general theories and experimental tests, a very broad view is presented. The mathematics associated with general relativity is often a challenge to students, and in the main text the mathematics is kept to a minimum. There is, however, a sound mathematical underpinning provided by the outline derivations of important results that are inserted into the main text.

For undergraduates with a previous knowledge of special relativity, the book provides a route to general relativity, with the chapters on special relativity providing useful acclimatization to the style and philosophy before the general theory is attempted. Warren Perkins Department of Physics, University of Wales Swansea, UK.

\begin{tabular}{ll}
\hline Range & $\star \star \star$ \\
Depth & $\star \star$ \\
\hline Accuracy & $\star \star \star$ \\
Up-to-dateness & $\star \star \star$ \\
\hline Accessibility & $\star \star$ \\
Style &
\end{tabular}

\section{Companion to the Cosmos}

by John Gribbin

Little Brown: 1996. Pp. 504. \$29.95/Weidenfeld and Nicolson $£ 20$

"Cosmos" is just an alternative word for "Universe" but "Companion" gives little hint that Gribbin is presenting us with a 416-page encyclopaedia of modern astronomy and cosmology plus a 67-page chronology of the history of the subject.

Good astronomical encyclopaedias require affirmative answers to three questions: "Is it in?", "Does the entry make sense when you find it?" and "Is the new encyclopaedia so good you can throw away older ones?". Gribbin certainly makes sense. He writes with such admirable clarity and interest that it is difficult to resist the diverting temptation of looking up what he thinks about other things. He concentrates on the exciting ideas and the influential proponents of astronomy. There is bias towards cosmology. There are no entries on constellations or named stars. James Clerk Maxwell gets more lines than Mars. Anthropic principle is in, ephemeris is out.

You will really enjoy this new encyclopaedia. But keep the old ones.

David W. Hughes Department of Physics, University of Sheffield, UK

\begin{tabular}{ll}
\hline Range & $\star \star$ \\
Depth & $\star \star \star$ \\
Accuracy & $\star \star \star \star$ \\
Up-to-dateness & $\star \star \star \star$ \\
Accessibility & $\star \star \star$ \\
Style & $\star \star \star$
\end{tabular}

\section{Limb Regeneration}

by Panagiotis Tsonis

Cambridge University Press:1996. Pp.241. £55, $\$ 74.95$

Regeneration of vertebrate body parts is frustratingly limited, but a few amphibians possess quite spectacular powers and can replace an entire limb. An understanding of how this happens would have important medical implications. Limb regeneration probably involves the reawakening of many of the processes used in the development of the embryonic limb. Molecular advances in the past five years have revolutionized knowledge of limb development, and this can now be applied to regeneration.

Unfortunately this book does not give this impression, and possible new directions exploring the roles of Sonic hedgehog, bone morphogenetic proteins and many more molecules now known to be important in limb development - are hardly mentioned. On the other hand, major issues in limb regeneration are set out well, and the review of traditional studies will prove useful to those not in the field.

Konstandina Kostakopoulou and Cheryll Tickle Department of Anatomy and Developmental Biology, University College London, UK

Range
Depth
Accuracy
Up-to-dateness
Accessibility
Style

$\star \star$
$\star \star$
$\star \star \star$
$\star \star \star$
$\star \star$
$\star \star \star$
$\star \star \star$
$\cdots$

